\title{
Relationship between microRNA-206 plasma levels with the severity of coronary artery conflicts in patients with coronary artery disease
}

\author{
Zehtabian $\mathrm{SH}^{1}$, Alibakhshi $\mathrm{R}^{1}$, Seyedena $\mathrm{SY}^{2}$, Rai $\mathrm{AR}^{3}$ \\ Department of biology, faculty of Biological science, Islamic Azad University, North Tehran Branch, \\ Tehran, Iran. ralibakhshiy@gmail.com
}

\begin{abstract}
OBJECTIVES: The aim of this study was to evaluate the possible association of miR-206 serum as an indicator of diagnosis in patients with coronary artery disease.

METHODS: In this study, 100 patients with coronary artery disease who had angiography and vascular transplantation were selected and evaluated. Extraction of microRNAs from peripheral blood plasma was performed using an exclusive microRNA extraction kit. Then the cDNA synthesis of the target microRNA was performed and its concentration and purity were evaluated. The expression level of miR-206 was performed using the real-time PCR technique and the SYBER Green method, using U6 snRNA as an internal control. In order to analyze the amount of microRNA expression and the significance of the patient sample, the t-test was used to compare the control sample. Also, Pearson correlation coefficient test was used to determine the relationship between the expression level of microRNAs.

RESULTS: The results showed a positive correlation between miR-206 expression and coronary artery disease. While the average expression of $1 \pm 0.18$ in the control sample was increased to 8.76 according to the severity of involvement in the patient, the relative expression of miR-206 in the CAD + group was significantly increased compared to the control $(p<0.03)$.

CONCLUSIONS: It appears that miR-206 can be considered as an indicator of coronary endothelial cell function. As such, it can be used as a biomarker for prognosis and in controlling the treatment for coronary artery disease (Tab. 2, Fig. 3, Ref. 20). Text in PDF www.elis.sk.

KEY WORDS: miR-206, coronary artery disease, real time PCR.
\end{abstract}

\section{Introduction}

Coronary artery disease (CAD) refers to the narrowing or obstruction of one or all parts of the coronary arteries due to the process of atherosclerosis or presence of a clot $(1,2)$. There are several ways to diagnose, detect, track and control cardiovascular treatment. In this regard, a variety of measures can be considered, namely the use of anticoagulants, achievement of PCI and coronary stenting, coronary artery bypass graft (CABS), and transplantation of parts of the saphenous vein. These treatments are invasive, dangerous and costly $(3,4)$. Therefore, it seems necessary to search for new methods for identifying individuals susceptible to stenosis.

In this regard, a study was conducted on microRNAs, a class of small regulatory RNAs important in regulating the expression

${ }^{1}$ Department of Biology, Faculty of Biological Science, Islamic Azad University, North Tehran Branch, Tehran, Iran, ${ }^{1 *}$ Department of Medical Genetics, Kermanshah University of Medical Sciences, Iran, ${ }^{2}$ Department of Biology, Faculty of Biological Science, Islamic Azad University, North Tehran Branch, Tehran, Iran, and ${ }^{3}$ Research \& Educational Center, Imam Ali Cardiovascular Hospital, Kermanshah University of Medical Sciences, Iran

Address for correspondence: R. Alibakhshi, Department of Medical Genetics, Kermanshah University of Medical Sciences, Iran.

Phone: +98.8334274618 , Fax: +98.8334276477 of genes (5). MicroRNAs are non-coding RNAs with a length of 19-24 nucleotides that regulate the expression of target cell mRNAs and are therefore involved in various cellular bioassays, including differentiation, proliferation, apoptosis, metabolism, regulation of gene expression and even neoplasia $(6,7)$.

In the case of microRNA-206, several studies have been conducted on its function in cardiac disorders $(8,9)$. MiR-206 regulates the activity of cells by directly affecting the protein kinase PIK3C2a and decreasing the expression of this protein kinase in endothelial progenitor cells. MiR-206 suppresses endothelial cell angiogenic activity and affects the angiogenic signal regulators such as Akt and the nitric oxide synthesis (10). In addition, miR206 has been shown to regulate and reduce the endothelial growth factor by reacting directly with the 3UTR of the VEGF gene, thus acting as an anti-VEGF agent. Hence, the increased expression of miR-206 in endothelial progenitor cells resulted in a specific reduction in endothelial growth factor, leading to a reduction in the progression of coronary artery disease. In this regard, the role of miR-206 as an important biomarker in angiogenesis lies also in the formation of vascular system. The role of this microRNA in the process of vasculogenesis where progenitors of endothelial cells of the vessels (angioblasts) differentiate and dilate to form primary blood vessels appears to be very important (11). 
$581-585$

Other analyses focusing on the role of this microRNA in the process of atherosclerosis revealed that miR-206 reduced the proliferation of smooth muscle cells by reacting with FoxP1. The FoxP1 factor as part of the FoxP family is a factor involved in transcription. FoxP1 is also associated with the interrelationship of interleukins 2, 4 and 10, which are involved in the stability of vascular platelets. As a result, the study found that miR-206 reduces the risk of atherosclerosis by reducing the proliferation and apoptosis of smooth muscle cells through the effect of FoxP1 (12).

In another study, the critical role of micro-RNA-206 in ischemic injury and myocardial reperfusion was investigated. Results revealed that the expression level of miR-206 was significantly decreased both in rat group and H9c2 cells subjected to hypoxia/ reoxygenation. Overexpression of miR-206 observably inhibited the cardiomyocyte apoptosis induced by injury. The expression of pro-apoptotic-related genes such as p53, Bax and cleaved caspase 3 was decreased in association with the downregulation of Gadd $45 \beta$. This demonstrates that miR-206 could protect against myocardial injury by targeting Gadd $45 \beta$ (13).

In more researches, miR-206 is an upstream regulator of $\mathrm{Cx} 43$ in cardiomyocytes, and its overexpression downregulates $\mathrm{Cx} 43$ to induce abnormal heart-rate. Connexin43 (Cx43) is an important cardiac gap junction protein and a potential target of miR-206, while downregulation of $\mathrm{Cx} 43$ induces ventricular tachyarrhythmias. MiR-206 directly recognized 3'-untranslated region of Cx43 mRNA to inhibit its expression. The induction of miR-206 in an adult mouse heart suppressed $\mathrm{Cx} 43$ expression, particularly in the atria and ventricle. Importantly, miR-206 overexpression also induced abnormal heart-rate, and shortened life-span (14).

In another study focused on HSP-60, an important component of the cell defense system in myocardial injury, miR-206 upregulates the expression of HSP-60 after the transplantation process, and in this way accelerates the apoptosis of cardiomyocytes. This regulatory process has been decreased and negatively influenced by the direct reaction of miR-206 with the 3UTR-mRNA of the HSP-60 gene, and consequently affected the expression of this gene (15). MiR-206 also controls and suppresses the level of IGF-1 in response to the 3UTR-mRNA region of IGF-1 and posttranscription regulation, and ultimately captures the apoptosis of the myoblast cells (16).

According to the above and in order to find potential biomarkers for prognosis, primary diagnosis, tracking treatment, and determining the stage of CAD, it seems necessary to research and determine the relationship between the level of expression of this microRNA and coronary artery bypass graft, as well as risk factors involved in CAD patients and healthy subjects.

\section{Materials and methods}

\section{Study population}

This research was conducted on patients who have been referred for angiography (PCI) and coronary artery bypass graft (CABS) at Imam Ali's cardiac and vascular research center in Kermanshah, Kurdish Region and West of Iran from February 16, 2017 , to December 2018. This study was performed on a case group of 100 patients with coronary artery disease and coronary artery stenosis with varying degrees of vascular involvement, (CAD + ) and a control group (CAD-) of 30 cases without coronary artery stenosis (CAD-). All blood samples from CAD+ patients are collected in nuclease-free tubes containing sterile EDTA anticoagulant (ethylene diamine tetraacetic acid). Samples were immediately stored at $-80{ }^{\circ} \mathrm{C}$ until tested. In addition, the demographic characteristics of the patients were registered.

\section{Extracting microRNAs \& primer design}

Extraction of microRNAs was carried out using a peripheral blood plasma sample, in which case the Favorgen miRNA isolation kit (Cat No: FAMIK001), capable of extracting small RNAs of less than 200 bp (including microRNAs), was used. This method is based on the selectable coupling of RNA molecules of different sizes to fiber matrix based on a silica-based fiber matrix in the presence of a chaotropic salt at different times. The purity and concentration of these microRNAs were measured using a spectrophotometer ultraviolet in $260 \mathrm{~nm}$ and $280 \mathrm{~nm}$ absorption using a US Nanodrop spectrophotometer Bio Tek. RNA samples showed high purity $>1.9$ at A260 / 280. The microRNA was then stored at $-80{ }^{\circ} \mathrm{C}$ up to the cDNA synthesis stage. An NCBI nucleotide database was used to have a favorable primer whose target areas had the least similarity with other sequences. Then, the protected areas were determined by multiple sequence alignment and MEGA4 software, and finally primers were designed using the Allel ID7 software.

The sequence used for miR-206 includes primers as follows: Forward: 5'-CCAAAG CGG AGT CTC GCAT-3' \& Reverse: 5'GCC TAG CAT CTT GCT TAG CTC-3' while that for U6 snRNA as housekeeping gene includes primers as follows: Forward: 5'CTC GCT TCG GCA GCA CA-3' \& Reverse Sequence: 5' - TGG TGT CGT GGA GT-3'.

\section{Synthesis of cDNA from MicroRNAs}

Synthesis of cDNA from microRNA was performed in two steps. The first step involves adding poly A tail to the $3^{\prime}$ end of microRNAs by a poly-A polymerase enzyme. The polyadenylation reaction consists of $10 \mathrm{X}$ buffer, poly A enzyme, ATP, and RNA with final volume of $20 \mu \mathrm{l}$ and then the reaction is incubated at $37^{\circ} \mathrm{C}$ for 10 minutes. In the next step, the synthesis of cDNA was performed using two primers, one reverse primer oligo dT-VN as primer adapter (Qiagen) and another direct primer as specific primer similar to the microRNA sequence (Qiagen). For this purpose, the dNTP mix, (M-MLV) RT enzyme, RNase inhibitor, DEPC water, and the corresponding buffer were used. Eventually, the single-stranded DNA of the microRNA supplement is made up to the final volume of $20 \mu \mathrm{l}$ at $42^{\circ} \mathrm{C}$ for 60 minutes. After cDNA synthesis, it is necessary to adjust the temperature for 5 minutes at 95 ${ }^{\circ} \mathrm{C}$ to disable the RT enzyme, incubate and complete the reaction.

\section{Real-time PCR measurement}

In the next step, the polymerase technique is used in quantitative real-time PCR to measure the microRNA expression level using two primers. The forward primer is similar to the specific 
sequence of miR-206 and reverse complement of the unique Oligo dT-VN primer. Each sample was measured at $1 \mu \mathrm{l}$ from cDNA to 10 ng concentration and duplicate in AB Applied Biosystems StepOneTM Real-Time PCR. In order to normalize the assay at each stage, U6 snRNA was used.

The reaction to measure this miR-206 in the final volume of $20 \mu \mathrm{L}$ consists of $10 \mu \mathrm{l} \mathrm{SYBR}$ green master mix, including hot start Taq DNA polymerase, Mgcl2, dNTP, dUTP, and the corresponding buffer, $1 \mu \mathrm{l}$ forward primer, $1 \mu \mathrm{l}$ reverse primer, $8 \mu 1$ cDNA(diluted), $0.4 \mu 1$ dye fluorescence color ROX. It should be noted that for each sample, the duplicate was used.

The temperature schedule for the SYBR green method took plsce as follows: 10 minutes at $95^{\circ} \mathrm{C}$ for activation of the enzyme, and then 40 cycles of proliferation including 10 seconds at $95^{\circ} \mathrm{C}, 60$ seconds at $57^{\circ} \mathrm{C}$ for annealing, and 30 seconds at $72{ }^{\circ} \mathrm{C}$ for the extension.

In each run, a sample is evaluated as a negative control to determine the master mix contamination and internal and positive controls. Optical data from the device entered the LinReg PCR software, version 11.Then for each sample (inclusive: internal control, normal and target) amplification Plot, CT (the cycle that interrupts the curve of the threshold line), $\Delta \mathrm{CT}$ (Ct difference between the specimen desired and internal control), $\Delta \Delta \mathrm{CT}$ ( $\Delta \mathrm{CT}$ difference between specimen or control patient and caliber samples), fold change (2 $-\Delta \Delta \mathrm{CT})$, melting curve and mean PCR efficiency were determined. Finally, based on the Livak's formula, the expression ratio of miR-206 in the patient sample was determined in comparison with the normal sample.

\section{Statistical analysis}

The data were analyzed by SPSS, vol.16 software after entering the computer. And the diagnostic value of serum microRNA was found to differentiate patients from control subjects. The data analysis was performed using Pearson correlation analysis to de-

Tab. 1. The expression level of miR-206 among the four CAD+ patient groups.

\begin{tabular}{lcc}
\hline $\begin{array}{l}\text { Type of coronary } \\
\text { artery disease }\end{array}$ & $\begin{array}{c}\text { Expression level } \\
\text { of miR-206 }\end{array}$ & $\mathrm{p}$ \\
\hline 1CAD & 3.08 & $<0.012$ \\
2CAD & 5.64 & $<0.022$ \\
3CAD & 8.14 & $<0.032$ \\
MCAD & 8.76 & $<0.035$ \\
Healthy control & $1 \pm 0.18$ & - \\
\hline
\end{tabular}

1CAD - one coronary artery involved; $2 \mathrm{CAD}$ - two coronary arteries involved; $3 \mathrm{CAD}$ - three coronary arteries involved; MCAD - main coronary artery involved

Tab. 2. Comparison of biochemical data among the CAD + patients.

\begin{tabular}{lccc}
\hline Factors & CAD + & CAD - & $p$ \\
\hline Sex $($ male/female $)$ & $87 / 13$ & $16 / 14$ & - \\
Age $($ year $)$ & $57 \pm 9$ & $55 \pm 8$ & 0.364 \\
BMI $\left(\mathrm{kg} / \mathrm{m}^{2}\right)$ & $27.78 \pm 3.45$ & $27 / 45 \pm 2.09$ & 0.228 \\
TC $(\mathrm{mg} / \mathrm{dl})$ & $190 \pm 45$ & $153 \pm 22$ & 0.407 \\
LDL $(\mathrm{mg} / \mathrm{dl})$ & $129 \pm 31$ & $100 \pm 13$ & 0.733 \\
HDL $(\mathrm{mg} / \mathrm{dl})$ & $33 \pm 3$ & $30 \pm 2$ & 0.278 \\
TG(mg/dl) & $188 \pm 49$ & $145 \pm 18$ & 0.320 \\
\hline BMI - body mass index; & TC - total cholesterol;
\end{tabular}

BMI - body mass index; TC - total cholesterol; LDL - low density lipoprotein; $\mathrm{HDL}$ - high density lipoprotein termine the relationship between miR-206 expression and severity of coronary artery disease. The t-test was also used to determine the significance of expression of miR-206 in the four groups involved in coronary artery disease, and a $p$ value less than 0.05 was considered statistically significant.

\section{Results}

Levels of expression of miR-206 in all patient samples (CAD+) showed a significant increase in expression in comparison with the healthy group (CAD-). With a significant difference (moderate mean of $p<0 / 025)$ in terms of expression level, this microRNA exists between negative and positive people (Tab. 1).

Also, according to the results, in $n=100$ patients, the expression of miR-206 was significantly and directly related to the percentage and number of vessels involved, indicating that the expression of miR-206 on the basis of coronary artery disease increased from 3.08 to 8.14 , as compared to the control group $(1 \pm 0.18)$

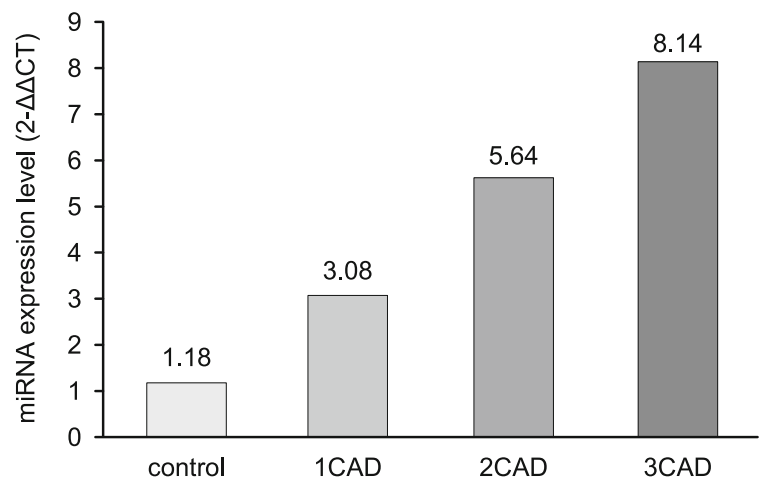

Fig. 1. The relative expression level of miR-206 per number of coronary arteries involved. 1CAD: one coronary artery involved; 2CAD: two coronary arteries involved; 3CAD: three coronary arteries involved; MCAD: main coronary artery involved.

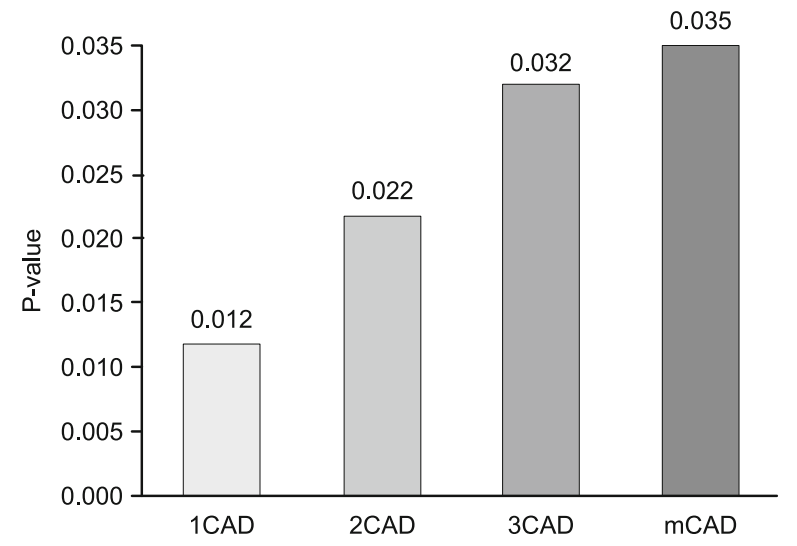

Fig. 2. Distribution of $P$ values and the significant level of clinical data of cardiovascular patients based on the number of vessels involved. 1CAD: one coronary artery involved; 2CAD: two coronary arteries involved; 3CAD: three coronary arteries involved; MCAD: main coronary artery involved. 


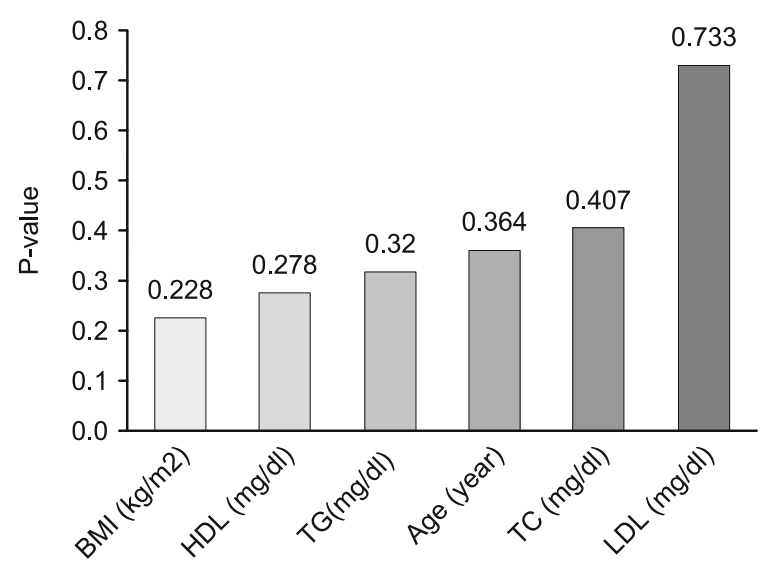

Fig. 3. Distribution of $P$ values and the significant level of biochemical variables and their correlation with coronary artery disease.

Of the total CAD + patients, 28 subjects were with one coronary arterial involvement, 17 patients with two coronary arterial involvement, 52 patients with three coronary arterial involvements and 3 patients had major coronary artery stenosis. In patients with one coronary artery involved, the serum level of expression of miR206 was 3.08 with a confidence interval of $0.91-2.51$ and mean age of 58 years. In those with two coronary arteries involved, the mean of serum miR-206 expression was 5.64, with confidence interval of $1.16-3.33$ and an average age of 60 years. In cases with three coronary arteries involved, the mean of serum miR-206 expression was 8.14 and with confidence interval of 1.31-3.97 and average age of 65 years. In patients with major coronary artery stenosis, the serum level of miR-206 was 8.76 with confidence level of 1.77-4.53 and mean age of 64 years. From the statistical point of view, Pearson's correlation coefficient is significant between groups $(p=0.01)$.

From the perspective of biochemical variables, the mean cholesterol level in the control group (CAD -) was $153 \pm 22$ and in the patient group $(\mathrm{CAD}+)$, it was $190 \pm 45$. Despite increasing cholesterol, the results of t-test showed that mean serum cholesterol levels yielded no significant difference between the studied groups $(\mathrm{p}<407)$.

Mean serum LDL in the control group (CAD -) was $100 \pm 13$ while in the patient group $(\mathrm{CAD}+)$, it was $129 \pm 31$. The results of t-test showed that mean serum LDL yielded no significant difference between the studied groups $(\mathrm{p}<0.733)$.

The mean serum HDL in the control group (CAD-) was $30 \pm 2$ and in the patient group $(\mathrm{CAD}+)$, it was $33 \pm 3$. The t-test showed that mean serum HDL yielded no significant difference between the studied groups $(\mathrm{p}<0.273)$.

Mean serum triglyceride was $145 \pm 18$ in the control group $\left(\mathrm{CAD}^{-}\right)$and $188 \pm 49$ in the case group $(\mathrm{CAD}+)$. The results of T-test showed that mean serum triglyceride levels yielded no significant difference between the studied groups $(\mathrm{p}<0.320)$ (Figs 1, 2 and 3 ).

\section{Discussion}

Over the past few years, researchers have been focusing on the role of microRNAs in cellular, molecular, and various disorders and diseases $(17,18)$.
The purpose of this study was to investigate the expression and role of microRNA-206 in cardiovascular disorders with coronary artery bypass grafting, Of course, the overall goal is to use this microRNA as a biomarker of the diagnostic function of these vessels and to control the treatment of this type of heart disease.

The precise method for checking this category of microRNAs is to use the SYBR green stain, which has the ability to bind to two-stranded DNA, provide a suitable fluorescence signal, and show the proliferation during real-time PCR $(19,20)$.

Recent research revealed different expressions of microRNA-206 in the control group (CAD-) and patient group (CAD +). The degree of expression varied in terms of pathological outcome of coronary artery disease (the percentage of obstruction to the number of vessels involved). In this regard, Pearson correlation analysis was performed to investigate the relationship between miR-206 expression and coronary artery disease. This showed the correlation coefficient to range between 0.75 and 0.89 , which is considered to be a positive correlation between the increase in the severity of vascular lesions and increase in miR-206. Which means that the increase in the expression of microRNA has a significant and direct correlation with the severity of coronary artery disease. At the same time, the t-test for determining the significance of miR-206 expression in the four groups of coronary artery disease showed a mean of $p<0 / 025$, which is a statistically significant difference between the two variables. With the exception of HDL, which shows a decrease and inverse correlation, a positive correlation and increase in specific expression was revealed also in important risk factors routinely evaluated in cardiovascular patients, including total cholesterol, LDL, TG, body mass index, and age. However, despite the fact that these routine parameters were increased in the four groups of patients, (with the involvement of one, two, three and major coronary arteries) this increase does not show a significant meaning. Perhaps it can be concluded that, despite the relative increase in this small range of routine factors, coronary artery lesions can be observed. Therefore, despite the increase in the level of expression of most parameters in this category of patients, the variables studied in this research, seem to be in a significant positive correlation with miR-206 biomarker.

\section{Conclusions}

The increase and specific change in miR-206 in coronary artery lesions may well reflect the changes in the endothelial function of coronary arteries in these patients. At the same time, this microRNA can be considered as a potential biomarker for detecting, tracking and controlling the status of coronary artery endothelial cells in this category of patients. Nevertheless, the more important point is that the expressive and meaningful expression of miR-206 may be an important indication for the diagnosis before any pathological clinical symptoms appear in cardiac patients.

Hence doing more research to reach the collection of microRNA panels accompanied by miR-206 can help us in the early detection of this type of cardiovascular disorder. 


\section{References}

1. Kanuri SH, Ipe J, Kassab K, Gao H. Next generation MicroRNA sequencing to identify coronary artery disease patients at risk of recurrent myocardial infarction. Atherosclerosis 2018 3; 278: 232-239.

2. Mozaffarian EJ, Benjamin AS. Heart disease and stroke Statistics-2016 update: a report from the American heart association. Circulation 2016; 133: e38-360.

3. Albus C, Barkhausen J. The Diagnosis of Chronic Coronary Heart Disease. Dtsch Arztebl Int 2017; 114 (42): 712-719.

4. Catalanotto C, Cogoni C. MicroRNA in Control of Gene Expression: An Overview of Nuclear Functions. Int J Mol Sci 2016; 17 (10): 1712.

5. Liu HG, Lei C, He Q. Nuclear functions of mammalian MicroRNAs in gene regulation, immunity and cancer. Molecular Cancer2018; 17: 64.

6. Mihailescu R. Gene expression regulation: lessons from noncoding RNAs. RNA 2015; 21 (4): 695-696.

7. Shan ZX, Lin QX, Deng CY, Zhu JN, Mai LP, Liu JL, Fu YH, Liu XY. miR-1/miR-206 regulate Hsp60 expression contributing to glucosemediated apoptosis in cardiomyocytes. FEBS Lett 2010; 584 (16): 3592 600 .

8. Shan ZX, Lin QX, Fu YH, Deng CY, Zhou ZL, Zhu JN, Liu XY, Zhang YY. Upregulated expression of miR-1/miR-206 in a rat model of myocardial infarction. Biochem Biophys Res Commun 2009; 381 (4): 597-601.

9. Tang Y, Zhang Y, Chen Y. Role of the miR-206 and its target PIK $3 \mathrm{C} 2 \alpha$ endothelial progenitor cell function - potential link with coronary artery disease. FEBS J 2015; 282 (19): 3758-3772.

10. Wang MJ, Ji Y, Cai SL. MiR-206 Suppresses the Progression of Coronary Artery Disease by Modulating Vascular Endothelial Growth Factor (VEGF) Expression. Med Sci Monit 2016; 22: 5011-5020.
11. Xing T, Du L, Zhuang X. Upregulation of microRNA-206 induces apoptosis of vascular smooth muscle cells and decreases risk of atherosclerosis through modulating FOXP1. Exp Ther Med 2017; 14 (5): 4097-4103.

12. Zhai C, Qian Q, Tang G. MicroRNA- 206 Protects against Myocardial Ischaemia-Reperfusion Injury in Rats by Targeting Gadd45 $\beta$. Mol Cells 2017; 40 (12): 916-924.

13. Jin Y, Zhou TY, Cao JN. MicroRNA-206 Downregulates Connexin43 in Cardiomyocytes to Induce Cardiac Arrhythmias in a Transgenic Mouse Model. Heart Lung Circ 2018; (18): 31917-31916.

14. Shan ZX, Lin QX, Deng CY, Zhu JN, Mai LP, Liu JL, Fu YH, Liu XY. miR-1/miR-206 regulate Hsp60 expression contributing to glucosemediated apoptosis in cardiomyocytes. FEBS Lett 2010; 584 (16): 35923600 .

15. Shan ZX, Lin QX, Fu YH, Deng CY, Zhou ZL, Zhu JN, Liu XY, Zhang YY. Upregulated expression of miR-1/miR-206 in a rat model of myocardial infarction. Biochem Biophys Res Commun 2009; 381 (4): 597-601.

16. Eichner NZM, Erdbrügger U. Extracellular Vesicles: A Novel Target for Exercise-Mediated Reductions in Type 2 Diabetes and Cardiovascular Disease Risk. J Diabetes Res 2018; 2018: 7807245.

17. Orlicka-Plocka M, Gurda D. Circulating microRNAs in Cardiovascular Diseases. Acta Biochim Pol 2016; 63 (4): 725-729.

18. Tien WP, Lim G. SYBR green-based one step quantitative real-time polymerase chain reaction assay for the detection of Zika virus in fieldcaught mosquitoes. Parasit Vectors 2017; 10: 427.

19. Farignoli M, Marciel W. Evaluation and optimization of SYBR Green real-time reverse transcription polymerase chain reaction as a tool for diagnosis of the Flavivirus genus in Brazil. Rev Soc Bras Med 2016; 167-198.

Received April 8, 2019. Accepted May 17, 2019. 